\title{
A METHOD OF SOLUTION OF THE EQUATIONS OF CLASSICAL GAS-DYNAMICS USING EINSTEIN'S EQUATIONS*
}

\author{
BY \\ G. C. McVITTIE \\ University of Illinois Observatory, Urbana, Ill.
}

Summary. It is known that Einstein's equations in general relativity provide explicit expressions for the density, pressure and velocity of a perfect gas in terms of the coefficients of the metric (the potentials) and hence in terms of the coordinates. Using orthogonal space-times, the expressions involve four potentials only between which consistency relations hold. It is shown how degeneration of the Einstein equations to Newtonian hydrodynamics provides general solutions of the equations of classical gas-dynamics for motions which may be either of constant or of variable entropy. The consistency relations are obtained in the general case. As an illustration, onedimensional gas-dynamics are discussed and it is shown how the consistency relations are manipulated. The solution in which one or other of the Riemann variables is constant is obtained as a special case and motions of variable entropy are also attained.

1. Introduction. It is well-known that Einstein's theory of general relativity is a generalization of the Newtonian mechanics of a continuous fluid but, as far as the present author is aware, it has not hitherto been realised that Einstein's theory can serve as a tool in classical gas-dynamics. The object of the present paper is to show how this comes about. The solutions of the equations governing the motion of a gas which are obtained impose no limitations on the magnitude of the gas-velocity nor do they pre-suppose that adiabatic conditions prevail. But they do imply that the gas is perfect and non-viscous and that its motion takes place under the influence of its pressuregradient alone.

The Newtonian absolute time will be denoted by $T$ and the rectangular coordinates in Newtonian absolute space by $\left(X_{1}, X_{2}, X_{3}\right)$. The three velocity components of the gas will be written $\left(U_{1}, U_{2}, U_{3}\right)$. The summation convention will be used throughout, repeated greek indices running through the values 1 to 4 , whilst repeated latin indices will take the values 1 to 3 . The letters $l, m, n$ will stand for any cyclic permutation of the numbers $1,2,3$. With these conventions, the classical equations of motion of a gas are

$$
\frac{\partial U_{i}}{\partial T}+U_{i} \frac{\partial U_{i}}{\partial X_{i}}=-\frac{1}{\rho} \frac{\partial p}{\partial X_{i}}+F_{i}, \quad(i=1,2,3),
$$

where $\left(F_{1}, F_{2}, F_{3}\right)$ is the force per unit mass of gas, excluding the pressure-gradient force; and the equation of continuity is

$$
\frac{\partial \rho}{\partial T}+\frac{\partial \rho U_{i}}{\partial X_{i}}=0
$$

The equations of motion can, with the aid of the continuity equation, also be written as

$$
\frac{\partial}{\partial T}\left(\rho U_{i}\right)+\frac{\partial}{\partial X_{i}}\left(\rho U_{i} U_{i}+\delta_{i j} p\right)=\rho F_{i}, \quad(i=1,2,3)
$$

*Received January 9, 1953. 
where $\delta_{i j}$ is the Kroenecker delta. The four equations (1.01) and (1.02) express the conservation of momentum and of mass in classical gas-dynamics. The thermodynamical quantities usually associated with a gas-motion are the velocity of sound, $a$, and the entropy of unit mass of gas, which may be defined as follows: If the specific heats at constant pressure and at constant volume are $c_{p}$ and $c_{v}$, respectively, and their ratio, $c_{p} / c_{v}$, is denoted by $k$, then

$$
a^{2}=k \frac{p}{\rho},
$$

The entropy, $S$, of unit mass of a perfect gas, whose temperature is $\theta$ and whose equation of state is $p=R \rho \theta$, is most conveniently defined thus: Let $d Q / d T$ be the rate of change "following the motion" of the heat-content of unit mass of gas, then

$$
J \frac{d Q}{d T}=J c_{\mathrm{v}} \frac{d \theta}{d T}+p \frac{d}{d T}\left(\frac{1}{\rho}\right)
$$

whence, using the equation of state and the relations

$$
R=J\left(c_{p}-c_{v}\right)=(k-1) J c_{v},
$$

it follows that

$$
\frac{1}{\theta} \frac{d Q}{d T}=c_{v} \frac{d}{d T}(\log p-k \log \rho) .
$$

In thermodynamics, the differential of the entropy is defined by $d Q / \theta$, but, for our purposes, it is more convenient to write

$$
d S=d Q /(c, \theta) .
$$

Hence

$$
\frac{d S}{d T}=\frac{d}{d T}\{\log p-k \log \rho\}
$$

or

$$
S=\log \kappa+\log p-k \log \rho,
$$

where $\kappa$ is a constant, characteristic of each separate unit mass of gas. In particular, if all unit masses have the same constant value of $\kappa$, the pressure and density of the gas are related by the isentropic relation

$$
p=\kappa \rho^{k} .
$$

It is now necessary to summarize briefly the notions of general relativity which will be required*, but the reader who is not interested in this aspect of the matter may pass directly to Equations (2.06) to (2.09) and content himself with the process of verification described in the text immediately following these equations. In general relativity, an event is specified by four coordinates $\left(x^{4}, x^{1}, x^{2}, x^{3}\right)$ of which the first denotes the time, and the other three, the place, of the occurrence in question. The

${ }^{*}$ For a more detailed treatment see, e.g. R. C. Tolman Relativity, Thermodynamics and Cosmology, Clarendon Press, Oxford, 1934. 
events which, for example, constitute the history of the motion of a perfect gas are regarded as mapped in a four-dimensional Riemannian space-time whose metric is

$$
d s^{2}=g_{\mu \nu} d x^{\mu} d x^{\nu} .
$$

The velocity of the gas is represented by a four-dimensional vector $\left(u^{4}, u^{1}, u^{2}, u^{3}\right)$ satisfying the equation

$$
1=g_{\mu \nu} u^{\mu} u^{\nu},
$$

whilst the mechanical quantities that are the counterparts of those whose partial derivatives appear in the equations (1.01) and (1.02), are the components of the energytensor

$$
T^{\mu \nu}=\rho u^{\mu} u^{\nu}-g^{\mu \nu} \frac{p}{c^{2}}
$$

where $\rho, p$ are the (invariant) density and pressure, $g^{\mu \nu}$ are the contravariant components of the metrical tensor $g_{\mu \nu}$, and $c$ is the velocity of light. The energy-tensor and the metrical tensor are connected by the ten Einstein equations, viz.

$$
-8 \pi \gamma T_{\nu}^{\mu}=G_{v}^{\mu}-\frac{1}{2} \delta_{\nu}^{\mu} G
$$

where $\gamma$ is the constant of gravitation, $T_{\nu}^{\mu}=g_{\nu \lambda} T_{\mu \lambda}, G_{\nu}^{\mu}$ is the contracted Riemann-Christoffel tensor and $G$, the invariant curvature The last two tensors can be expressed in terms of the $g_{\mu \nu}$ and their first and second derivatives with respect to the roordinates, by the rules of the tensor calculus.

2. Newtonian approximation to Einstein's equations. It is sufficient for our purpose to consider orthogonal space-times whose coefficients differ only slightly from those of the space-time of special relativity where

$$
g_{44}=1, \quad g_{11}=g_{22}=g_{33}=-\frac{1}{c^{2}}, \quad g_{\mu \nu}=0 \quad(\mu \neq \nu) .
$$

We write $\epsilon=2 \gamma / c^{2}$ and assume throughout the calculations that terms of order $\epsilon$ higher than the first are negligible. An orthogonal space-time of the required kind has metric

$$
d s^{2}=D\left(d x^{4}\right)^{2}-A\left(d x^{1}\right)^{2}-B\left(d x^{2}\right)^{2}-C\left(d x^{3}\right)^{2}
$$

where

$$
\left.\begin{array}{l}
D=1-4 \pi \epsilon \varphi, \\
A=\frac{1}{c^{2}}\left\{1+4 \pi \epsilon\left(\varphi+\frac{2 \varphi_{1}}{c^{2}}\right)\right\} \\
B=\frac{1}{c^{2}}\left\{1+4 \pi \epsilon\left(\varphi+\frac{2 \varphi_{2}}{c^{2}}\right)\right\}, \\
C=\frac{1}{c^{2}}\left\{1+4 \pi \epsilon\left(\varphi+\frac{2 \varphi_{3}}{c^{2}}\right)\right\},
\end{array}\right\}
$$

and $\varphi, \varphi_{1}, \varphi_{2}, \varphi_{3}$ are functions of all four coordinates $\left(x^{4}, x^{1}, x^{2}, x^{3}\right)$. The right-hand sides of (1.10) have been calculated by Dingle* for general values of $D, A, B, C$. If terms 
of order $\epsilon$ are alone retained in his formulae, the following approximate forms of Einstein's equations are obtained:

$$
\left.\begin{array}{l}
4 \pi \epsilon T^{l m}=-4 \pi \epsilon \frac{\partial^{2} \varphi_{n}}{\partial x^{l} \partial x^{m}}, \\
4 \pi \epsilon T^{l 4}=4 \pi \epsilon \frac{\partial^{2}}{\partial x^{l} \partial x^{4}}\left\{\varphi+\frac{1}{c^{2}}\left(\varphi_{m}+\varphi_{n}\right)\right\}, \\
4 \pi \epsilon T^{l l}=4 \pi \epsilon\left\{-\frac{\partial^{2}}{\left(\partial x^{4}\right)^{2}}\left(\varphi+\frac{\varphi_{m}+\varphi_{n}}{c^{2}}\right)+\frac{\partial^{2} \varphi_{m}}{\left(\partial x^{n}\right)^{2}}+\frac{\partial^{2} \varphi_{n}}{\left(\partial x^{m}\right)^{2}}\right\}, \\
4 \pi \epsilon T^{44}=-4 \pi \epsilon\left\{\nabla^{2} \varphi+\frac{1}{c^{2}} \sum_{l, m, n}\left(\frac{\partial^{2}}{\left(\partial x^{m}\right)^{2}}+\frac{\partial^{2}}{\left(\partial x^{n}\right)^{2}}\right) \varphi_{l}\right\},
\end{array}\right\}
$$

where

$$
\nabla^{2}=\frac{\partial^{2}}{\left(\partial x^{1}\right)^{2}}+\frac{\partial^{2}}{\left(\partial x^{2}\right)^{2}}+\frac{\partial^{2}}{\left(\partial x^{3}\right)^{2}} .
$$

Using also (1.09), the preceding equations become

$$
\left.\begin{array}{rl}
\rho u^{l} u^{m} & =-\frac{\partial^{2} \varphi_{n}}{\partial x^{l} \partial x^{m}}, \\
\rho u^{l} & =\frac{\partial^{2}}{\partial x^{4} \partial x^{2}}\left(\varphi+\frac{\varphi_{m}+\varphi_{n}}{c^{2}}\right), \\
\rho\left(u^{l}\right)^{2}+p & =-\frac{\partial^{2}}{\left(\partial x^{4}\right)^{2}}\left(\varphi+\frac{\varphi_{m}+\varphi_{n}}{c^{2}}\right)+\frac{\partial^{2} \varphi_{m}}{\left(\partial x^{n}\right)^{2}}+\frac{\partial^{2} \varphi_{n}}{\left(\partial x^{m}\right)^{2}}, \\
\rho\left(u^{4}\right)^{2}-\frac{p}{c^{2}} & =-\nabla^{2} \varphi-\frac{1}{c^{2}} \sum_{l, m, n}\left\{\frac{\partial^{2}}{\left(\partial x^{m}\right)^{2}}+\frac{\partial^{2}}{\left(\partial x^{n}\right)^{2}}\right\} \varphi_{l},
\end{array}\right\}
$$

where, to a sufficient approximation, the velocity four-vector satisfies

$$
\left(u^{4}\right)^{2}-\frac{1}{c^{2}}\left\{\left(u^{1}\right)^{2}+\left(u^{2}\right)^{2}+\left(u^{3}\right)^{2}\right\}=1 .
$$

There are eleven equations in the set (2.04) and (2.05), whose left-hand sides contain the six functions of the coordinates $\rho, p, u^{4}, u^{1}, u^{2}, u^{3}$; whilst their right-hand sides involve the four functions $\varphi, \varphi_{1}, \varphi_{2}, \varphi_{3}$. Obviously therefore there must be additional relationships between these two sets of functions and, in principle at least, it is possible to eliminate $\rho, p, u^{4}, u^{1}, u^{2}, u^{3}$ from (2.04) and (2.05) and thus obtain differential equations involving only $\varphi, \varphi_{1}, \varphi_{2}$, and $\varphi_{3}$. Such equations will be called consistency relations because, unless $\varphi, \varphi_{1}, \varphi_{2}, \varphi_{3}$ satisfy them, the eleven equations (2.04) and (2.05) will be mutually inconsistent. We shall show later how these consistency relations are obtained and how they are manipulated in the calculations: we must first derive from (2.04) and (2.05) the corresponding equations in Newtonian hydrodynamics. This is done, as usual in relativity theory, by neglecting terms of order $1 / c^{2}$ and, to this end, it will be assumed that $\varphi, \varphi_{1}, \varphi_{2}, \varphi_{3}$, and the four $u^{\alpha}$ contain no terms of order $c^{2}$. The 
Newtonian velocity components $\left(U_{1}, U_{2}, U_{3}\right)$ will be the degenerate forms of the ratios $\left(d x^{1} / d x^{4}, d x^{2} / d x^{4}, d x^{3} / d x^{4}\right)=\left(u^{1} / u^{4}, u^{2} / u^{4}, u^{3} / u^{4}\right)$ when terms of order $1 / c^{2}$ are neglected; and the coordinates $\left(x^{4}, x^{1}, x^{2}, x^{3}\right)$ will become the Newtonian coordinates $\left(T, X_{1}, X_{2}, X_{3}\right)$. Neglecting terms of order $1 / c^{2}$ equation (2.05) reduces to the statement that $u^{4}=1$ whilst the following set of equations is obtained from (2.04):

$$
\begin{aligned}
\rho U_{l} U_{m} & =-\frac{\partial^{2} \varphi_{n}}{\partial X_{l} \partial X_{m}}, \\
\rho U_{i} & =\frac{\partial^{2} \varphi}{\partial X_{i} \partial T}, \quad(i=1,2,3), \\
\rho U_{l}^{2}+p & =-\frac{\partial^{2} \varphi}{\partial T^{2}}+\frac{\partial^{2} \varphi_{n}}{\partial X_{m}^{2}}+\frac{\partial^{2} \varphi_{m}}{\partial X_{n}^{2},} \\
\rho & =-\nabla^{2} \varphi .
\end{aligned}
$$

In these ten equations $\rho, p$ now stand for the Newtonian density and pressure respectively. It is easy to show by direct partial differentiation that, if the quantities given by (2.06) to (2.09) be substituted into (1.01) and (1.02), these equations are satisfied identically provided that $F_{i}=0,(i=1,2,3)$. Thus a solution of the equations of classical gas-dynamics has been obtained for the case when the gas is moving under the action of its pressure-gradient alone. The solution however involves the four functions $\varphi, \varphi_{i}(i=$ $1,2,3)$ of the coordinates $X_{i}$ and of $T$, which are not independent but are subject to consistency relations that may be obtained as follows:

Elimination of $\rho$ and the three $U_{i}$ from equations (2.06), (2.07) and (2.09) yields the three equations

$$
\frac{\partial^{2} \varphi_{l}}{\partial X_{m} \partial X_{n}}=\left(\frac{\partial^{2} \varphi}{\partial X_{m} \partial T} \cdot \frac{\partial^{2} \varphi}{\partial X_{n} \partial T}\right) / \nabla^{2} \varphi .
$$

Again elimination of $\rho, p$ and the three $U_{i}$ from (2.07), (2.08) and (2.09) yields three equations, only two of which are however independent,

$$
\left(\frac{\partial^{2}}{\partial X_{l}^{2}}-\frac{\partial^{2}}{\partial X_{m}^{2}}\right) \varphi_{n}+\frac{\partial^{2}}{\partial X_{n}^{2}}\left(\varphi_{l}-\varphi_{m}\right)=\left\{\left(\frac{\partial^{2} \varphi}{\partial X_{l} \partial T}\right)^{2}-\left(\frac{\partial^{2} \varphi}{\partial X_{m} \partial T}\right)^{2}\right\} / \nabla^{2} \varphi .
$$

The ten equations (2.06) to (2.09) may then be replaced by the five consistency relations (2.10) and (2.11) together with the further five equations

$$
\left.\begin{array}{rl}
U_{i} & =-\frac{\partial^{2} \varphi}{\partial X_{i} \partial T} / \nabla^{2} \varphi, \quad(i=1,2,3), \\
\rho & =-\nabla^{2} \varphi, \\
p & =-\frac{\partial^{2} \varphi}{\partial T^{2}}+\frac{1}{3} \nabla^{2}\left(\sum_{i=1}^{3} \varphi_{i}\right)-\frac{1}{3} \sum_{i=1}^{3} \frac{\partial^{2} \varphi_{i}}{\partial X_{i}^{2}}+\frac{1}{3} \sum_{i=1}^{3}\left(\frac{\partial^{2} \varphi}{\partial X_{i} \partial T}\right)^{2} / \nabla^{2} \varphi
\end{array}\right\}
$$

where, in the formula for $p$, it is presumed that $\varphi_{1}, \varphi_{2}, \varphi_{3}$ have been chosen so as to satisfy the consistency relations. It is worth noticing that $\rho$ and $4 \pi \varphi$ are connected by Poisson's equation and therefore $4 \pi \varphi$ is the gravitational potential of the distribution of gas. The corresponding gravitational force has however been neglected in determining 
the motion of the gas since, as we have seen, equations (2.06) to (2.09) imply that no force other than the pressure-gradient is acting.

3. One-dimensional gas-dynamics. As an illustration of the foregoing general theory, we consider certain types of motion in which the velocity of the fluid is parallel to a given straight line and in which the pressure and density vary spatially only with respect to distance measured parallel to this line. If the line is chosen to be the $X_{1}$-axis and distance along it be denoted by $X$, motions of this kind are defined by assuming that all the variables in equations (2.10), (2.11) and (2.12) are functions of $X$ and $T$ alone. It then follows at once from (2.12) that $U_{2}=0, U_{3}=0$, and that only $U_{1}=U$ survives. The consistency relations (2.10) are identically satisfied, whilst the relations (2.11) are also satisfied by taking

$$
\left.\begin{array}{rl}
\varphi_{1} & =0, \quad \varphi_{2}=\varphi_{3}, \\
\frac{\partial^{2} \varphi_{2}}{\partial X^{2}} & =\left(\frac{\partial^{2} \varphi}{\partial X \partial T}\right)^{2} / \frac{\partial^{2} \varphi}{\partial X^{2}} .
\end{array}\right\}
$$

Hence formulae (2.12) reduce to

$$
\left.\begin{array}{rl}
U & =-\frac{\partial^{2} \varphi}{\partial X \partial T} / \frac{\partial^{2} \varphi}{\partial X^{2}}, \\
\rho & =-\frac{\partial^{2} \varphi}{\partial X^{2}}, \\
p & =-\frac{\partial^{2} \varphi}{\partial T^{2}}+\left(\frac{\partial^{2} \varphi}{\partial X \partial T}\right)^{2} / \frac{\partial^{2} \varphi}{\partial X^{2}},
\end{array}\right\}
$$

which provide the general solution of the problem of one-dimensional gas-dynamics which we are seeking by the method of Einstein's equations. The solution is given to within an arbitrary function $\varphi$ of $X$ and $T$, which, from the purely mathematical standpoint, can be chosen in any way we please. But it is important to notice that not every such selection will give a physically acceptable solution as may be seen by considering the choice

$$
\varphi=A \cos (X-q T),
$$

where $A, q$ are constants, which leads through (3.02) to

$$
U=q, \quad \rho=A \cos (X-q T), \quad p=0 .
$$

Thus the velocity of the gas is constant, its pressure is zero and its density is alternately positive and negative, vanishing at places where $X=q T \pm\left(n+\frac{1}{2}\right) \pi$, ( $n$, an integer). Such a distribution of gas would clearly not be considered physically admissible.

The method of solution of equations (3.02) therefore consists of finding, by trial and error, a function $\varphi$ that will correspond to a physically significant situation. The process of selection may be guided by some a priori requirement respecting the mathematical forms of $U, p$ or $\rho$. As an example, let it be required to find all permissible densities and pressures for a perfect gas moving in such a way that its velocity is given by

$$
U=(n+1)[X / T+n q /(n+1)],
$$


where $n$ is a pure number and $q$ is a constant with the dimensions of velocity. Writing $\mu=\partial \varphi / \partial X$ and substituting from (3.03) into the first of equations (3.02), it follows that $\mu$ satisfies the first-order partial differential equation

whence

$$
\frac{\partial \mu}{\partial T}+(n+1)\left(\frac{X}{T}+\frac{n q}{n+1}\right) \frac{\partial \mu}{\partial X}=0,
$$

$$
\mu=\frac{\partial \varphi}{\partial X}=f\left\{\left(\frac{X}{T}+q\right) T^{-n}\right\}
$$

where $f$ is an arbitrary function of the argument $(X / T+q) T^{-n}$. It then follows that

$$
\varphi=T^{n+1} F\left\{\left(\frac{X}{T}+q\right) T^{-n}\right\}+H(T),
$$

where $H$ is an arbitrary function of $T$ and

$$
F=\int f\left\{\left(\frac{X}{T}+q\right) T^{-n}\right\} T^{-(n+1)} d X,
$$

$T$ being treated as a constant in this integration Introducing a new variable $\zeta$ by

$$
\zeta=\left(\frac{X}{T}+q\right) T^{-n}
$$

and denoting differentiation with respect to $T$ by a prime, equations (3.02) yield

$$
\left.\begin{array}{l}
U=(n+1)\left\{\zeta T^{n}-q /(n+1)\right\}, \\
\rho=-T^{-(n+1)} \frac{d^{2} F}{\partial \zeta^{2}}, \\
p=n(n+1) T^{n-1}\left\{\zeta^{2} \frac{d}{d \zeta}\left(\frac{F}{\zeta}\right)-\frac{H^{\prime \prime} T^{1-n}}{n(n}+\frac{1)}{1)}\right\} .
\end{array}\right\}
$$

By means of these expressions for $p$ and $\rho$, it is possible to calculate the velocity of sound in the gas and the rate of change of entropy of unit mass following its motion. Formula (1.03) gives

$$
a^{2}=k(n+1) n\left\{T^{2 n} \zeta^{2} \frac{d}{d \zeta}\left(\frac{F}{\zeta}\right)-\frac{H^{\prime \prime} T^{1+n}}{n(n+1)}\right\} /\left(-\frac{d^{2} F}{d \zeta^{2}}\right),
$$

whilst (1.05) with $d / d T=\partial / \partial T+U(\partial / \partial X)$ yields

$$
\begin{aligned}
\frac{d S}{d T}= & \frac{k(n+1)}{T} \\
& +{ }^{n} \frac{-1}{T}\left\{\zeta^{2} \frac{d}{d \zeta}\left(\frac{F}{\zeta}\right)-\frac{H^{\prime \prime \prime} T^{2}}{(n+1) n(n}-1\right) / /\left\{\zeta^{2} \frac{d}{d \zeta}\left(\frac{F}{\zeta}\right)-\frac{H^{\prime \prime} T^{1-n}}{(n+1) n}\right\} .
\end{aligned}
$$

Obviously, a physically acceptable case must have $\rho>0$, i.e. $F$ must be such that $d^{2} F / d \zeta^{2}<0$ for all values of $\zeta$ corresponding to the region of space occupied by the moving gas, and for all relevant times. Moreover the pressure $p$ and the square of the velocity of sound must both be positive; hence $F, H$ and $n$ must be chosen so as to fulfill 
these requirements. As an aid to the selection of $F, H$ and $n$ it is instructive to specialize formula (3.08) in two independent ways. Firstly, let it be assumed that $H$ satisfies

$$
H^{\prime \prime}=(\text { constant }) T^{n-1} \text {, }
$$

where the constant may have the value zero. Equation (3.08) then reduces to

$$
\frac{d S}{d T}=\frac{k(n+1)+(n-1)}{T} .
$$

Alternatively, let it be assumed that each unit mass of gas conserves its entropy as it moves, a condition expressed by $d S / d T=0$ or

$$
\{k(n+1)+(n-1)\} \zeta^{2} \frac{d}{d \zeta}\left(\frac{F}{\zeta}\right)-\frac{T^{1-n}}{(n+1) n}\left\{k(n+1) H^{\prime \prime}+T H^{\prime \prime \prime}\right\}=0 .
$$

If both (3.09) and (3.11) hold simultaneously, and $F$ is arbitrary, $n$ is determined in terms of $k$ by

$$
n=-(k-1) !(k+1),
$$

and since for a real gas

$$
2>k>1
$$

$n$ must be a negative number.

(i) Velocity of Sound a Linear Function of $X / T$.

The effect of choosing particular mathematical expressions for $F$ must now be investigated and, as a first example, suppose that

$$
F=-A \zeta^{\lambda}
$$

where $A(>0)$ and $\lambda$ are constants; and suppose also that (3.09) is satisfied by taking $H \equiv 0$. Then (3.06) and (3.07) become

$$
\begin{aligned}
& U=(n+1)\left\{\zeta \zeta T^{n}-q /(n+1)\right\}=(n+1)\{X / T+n q /(n+1)\}, \\
& \rho=A \lambda(\lambda-1) \zeta^{\lambda-2} T^{-(n+1)}=A \lambda(\lambda-1)(X / T+q)^{\lambda}{ }^{2} T^{-n \cdot \lambda-1}, \cdots, \\
& p=-A(\lambda-1) n(n+1) \zeta^{\lambda} T^{n-1}=-A(\lambda-1) n(n+1)(X T+q)^{\lambda} T^{-n(\lambda-1}, 1, \\
& a=\left\{-\frac{k}{\lambda} n(n+1) \zeta^{2} T^{2 n}\right\}^{1 / 2}=\left\{-\frac{k}{\lambda} n(n+1)\right\}^{1 / 2}(X / T+q),
\end{aligned}
$$

whilst $d S / d T$ is given by (3.10). Thus the choice (3.14) for $F$ leads to an expression for the velocity of sound which, like that for $U$, is linear in $X / T$. But $p, \rho$ and $a^{2}$ must all be positive in a physically acceptable solution and therefore

$$
n(n+1)<0 \quad \text { and } \quad \lambda>1 \text {. }
$$

The first of these conditions is clearly satisfied in the constant entropy case in which $n$ is given by (3.12). If further, the motion is isentropic and the expressions (3.15) for $p$ and $\rho$ are substituted into (1.07), it follows. by considering the indices of $\zeta$ and $T$ 
in the resulting formula, that

$$
k(\lambda-2)=\lambda \quad \text { and } \quad k(n+1)=1-n .
$$

The second condition is, of course, the same as (3.12) whilst the first determines $\lambda$ in terms of $k$, viz.

$$
\lambda=2 k /(k-1) \text {. }
$$

Because of (3.13), $\lambda$ is greater than unity as required by (3.16). Using (3.12), (3.17) and (3.15), the solution for isentropic motion is

$$
\left.\begin{array}{rl}
U & =\frac{2}{k+1}\left(\frac{X}{T}-\frac{k-1}{2} q\right), \\
\rho & =\frac{2 k(k+1) A}{(k-1)^{2}}\left(\frac{X}{T}+q\right)^{2 /(k-1)}, \\
p & =\frac{2 A}{k+1}\left(\frac{X}{T}+q\right)^{2 k /(k-1)}, \\
a & =\frac{k-1}{k+1}\left(\frac{X}{T}+q\right) .
\end{array}\right\}
$$

In the conventional treatment of isentropic motion in gas-dynamics based on the method of Riemann*, the solution (3.18) is obtained by putting one of the Riemann variables $r, s$ equal to a constant. But the Riemann method gives, in the first instance, explicit values of $U$ and of $a$, whereas the present one simultaneously determines $p, \rho, U$ and $a$. This is due to the use of the gravitational potential $4 \pi \varphi$ that corresponds to the density $\rho$. A second point of difference is that the isentropic condition (1.07) is introduced a priori in Riemann's method and it is therefore not easy to modify the method when variable entropy motions are in question. Such cases arise, for example, in the motions of interstellar gas clouds which are losing energy by radiation, a problem that has been attempted by Burgers $\dagger$ using the classical treatment. Variable entropy motions however present no greater difficulty than do adiabatic motions if the method we are here presenting be employed. For example, the solution (3.15) with the conditions (3.16) corresponds in general to variable entropy, the rate of change of entropy following the motion falling off inversely with the time by (3.10). In such motions the rate of loss of internal heatenergy in ergs per sec. per $\mathrm{cm}^{3}$ of the gas is

$$
\frac{d E}{d T}=-J \rho \frac{d Q}{d T}=-\frac{R \rho \theta}{k-1} \frac{d S}{d T}=-\frac{p}{k-1} \frac{d S}{d T} .
$$

Using (3.10), and (3.15) there comes

$$
\frac{d E}{d T}=\frac{-A(\lambda-1)}{k-1}\{-n(n+1)\}\{k(n+1)+(n-1)\}\left(\frac{X}{T}+q\right)^{\lambda} T^{-n(\lambda-1)-2} .
$$

*B. Riemann, Oeuvres Mathématiques, Paris, p. 177, 1898. A summary of the method is given in G. C. McVittie, Mon. Not. Roy. Astron. Soc., London 110, 224-237, (1950).

$\dagger J$. M. Burgers, K. Ned. Akad. v. Wet., 29, 600 (1946). 
By hypothesis $A>0$, and, by (3.13) and (3.16), $2>k>1, \lambda>1$, and $-1<n<0$; hence all the constant factors in $d E / d T$ are positive provided that $k(n+1)+(n-1)$ is also positive. If the gas is monatomic, $k=5 / 3$, and $n$ must lie in the restricted range $-1 / 4<n<0$; if the gas is diatomic, $k=7 / 5$, and $n$ lies in $-1 / 6<n<0$; and so on. Thus by a suitable choice of $n$ depending on the value of $k$, equations (3.15) give the motion of a gas which is losing heat-energy per unit volume at the rate given by (3.19).

(ii) Velocity of Sound a Quadratic Function of $X / T$.

As a second illustrative example in the choice of $F$ and $H$, we consider

$$
F=-A \zeta \log (\zeta+B), \quad H^{\prime \prime}=4 A B n(n+1) T^{n-1}, \quad \frac{d S}{d T}=0,
$$

where $A(>0)$ and $B$ are constants. Equations (3.06) and (3.07) then become

$$
\begin{aligned}
U & =(n+1)\{X / T+n g /(n+1)\}, \\
\rho & =\frac{A}{T}\left(X, T+q+2 B T^{n}\right) /\left(X / T+q+B T^{n}\right)^{2}, \\
p & =-n(n+1) \frac{A}{T}\left(X / T+q+2 B T^{n}\right)^{2} /\left(X / T+q+B T^{n}\right), \\
a^{2} & =-n(n+1) k\left(X / T+q+2 B T^{n}\right)\left(X / T+q+B T^{n}\right),
\end{aligned}
$$

where $n=-(k-1) /(k+1)$. Since $n<0$ for any real gas, it is evident that, as $T$ increases, $U$ and $a$ will ultimately both be linear functions of $(X / T+q)$, as in (3.18), but the formulae for $\rho$ and $p$ will not become functions of $(X / T+q)$ alone.

4. Further developments and conclusions. The one-dimensional gas-dynamics discussed in the preceding section do not exhaust the applications of the equations (2.10), (2.11) and (2.12). The case of spherical masses of gas in motion has also proved tractable and, by proceeding to the second approximation to Einstein's equations through the inclusion of terms in $\epsilon^{2}$, it has been possible to take account of the gravitational self-attraction of the mass of gas. The initial coordinate system employed in (2.01) is moreover not unique and an analysis of the different permissible coordinate systems has thrown light on the meaning of coordinate systems in general relativity and on the problem of gravitational waves in that theory. These and other related problems will be discussed by the author in a forthcoming publication. In the meantime, it has seemed appropriate to give the essence of the method in the hope that it will be found useful by other investigators. 